\title{
Studies on Bioactive Actinomycetes in a Niche Biotope, Nambul River in Manipur, India
}

Debananda S. Ningthoujam*, Suchitra Sanasam and Salam Nimaichand

Microbial Biotechnology Biotechnology Laboratory (MBRL), Department of Biochemistry, Manipur University, Canchipur, Imphal 795003, India

\begin{abstract}
As part of our ongoing studies on actinomycete diversity in Manipur, an underexplored zone falling in the IndoBurma biodiversity hotspot, this paper reports bioactivity screening and characterization of bioactive actinomycetes from Nambul River. Bioprospecting studies on actinobacteria have been largely focused on terrestrial and, more recently, on marine ecosystems but freshwater habitats have been largely neglected and studies on freshwater actinomycetes are very scanty in India. Hence we investigated the actinomycete diversity in one of the freshwater rivers of Manipur, Nambul River in Manipur, India. A total of 156 actinomycetes were isolated from three samples of Nambul River. Based on the results of primary screening, 23 isolates were selected for secondary screening. Nine strains showed significant antibacterial or broad spectrum antimicrobial (antibacterial and antifungal) activities in the secondary screening. Phylogenetic analyses indicated that a majority of them were Streptomyces species though some rare actinobacteria were also recovered. Seven strains were identified as Streptomyces spp. while one strain each was identified as Nocardia sp. and Micromonospora sp. Three strains showed promising antifungal activities against human and plant pathogens. This study highlights the potential for discovering bioactive actinomycetes in underexplored niche biotopes such as river sediments.
\end{abstract}

Keywords: Nambul river; Bioactive; Antifungal; Novel species; Streptomyces

\section{Introduction}

Actinomycetes are a group of physiologically versatile, high GC, gram-positive, filamentous bacteria found in most environments including terrestrial and aquatic habitats [1]. Streptomyces has been reported as the dominant genus in freshwater habitats whereas Micromonospora and related genera are predominant in freshwater and marine sediments [2].

There is increasing realization of the potential for wetlands as sources of actinomycetes that produce useful bioactive compounds. Cross [3] reported freshwater habitats as promising sources of bioactive actinomycetes. Okami [4] reported that actinomycetes of freshwater origin produce novel bioactive substances. There is an urgent need for screening of novel bioactive compounds from underexplored biotopes such as freshwater habitats. This is also dictated by the rise of emerging diseases and antibiotic-resistant human pathogenic bacteria such as multidrug resistant (MDR) strains of $M$. tuberculosis, vancomycin resistant enterococci (VRE), methicilin resistant Staphylococus aureus (MRSA), Pseudomonas aeruginosa and Candida albicans [5] etc. The focus is increasing towards novel biotopes, niche ecosystems and extreme environments for isolating novel bioactive strains [6] especially actinobacteria which produce nearly $80 \%$ of all known antibiotics [7]. Additionally the microbial profiles also serve as an indicator of freshwater ecological health [8].

\section{Materials and Methods}

\section{Sampling and pretreatment}

Sampling was done from three different sites of the Nambul River, which is one of the major rivers in Manipur (62.7 km in length), originating from Kangchup Hill range in the western side at an elevation of $1830 \mathrm{~m}$ above mean sea level. The river flows through the thickly populated area of the city and ultimately discharges into the Loktak Lake. The potentially polluted stretch of the river is within the
Imphal Municipality area for a length of about $1.45 \mathrm{~km}$ and its tributary Naga Nala for a length of about $1 \mathrm{~km}$. Soils and sediment samples were collected from the Nambul river bank, river bed and the rhizospheric sediments of river water vegetation in polyethylene bags, closed tightly, and stored in a refrigerator before processing.

Pretreatment of the soil samples were carried out by air-drying them at room temperature for about four weeks $[9,10]$.

\section{Enrichment and isolation}

To further enrich the actinomycete population, $1.0 \mathrm{~g}$ air-dried sediment was mixed with $0.1 \mathrm{~g}$ of $\mathrm{CaCO}_{3}$ and kept at ambient temperature for a week to enrich actinomycetes which usually prefer alkaline conditions and also to reduce the contamination of molds and fungi [11]. $1.0 \mathrm{~g}$ air-dried sediment was suspended in $99.0 \mathrm{ml}$ of sterile distilled water and incubated in an orbital shaker at room temperature at $150 \mathrm{rpm}$ for 30 minutes. The soil suspension was then serially diluted and $0.1 \mathrm{ml}$ of $10^{-3}$ to $10^{-7}$ dilutions were spread plated in duplicates on Starch Casein Nitrate Agar (SCNA, pH 7.2) plates [12] supplemented with $50 \mu \mathrm{g} \cdot \mathrm{mL}^{-1}$ each of nystatin and cycloheximide [13] and finally incubated at $28^{\circ}-30^{\circ} \mathrm{C}$ for up to 4 weeks.

Selected actinomycete colonies were further purified on SCNA plates and pure isolates were maintained on modified Bennett's agar

*Corresponding author: Debananda S. Ningthoujam, Microbial Biotechnology Biotechnology Laboratory (MBRL), Department of Biochemistry, Manipur University, Canchipur, Imphal 795003, India, E-mail: debananda.ningthoujam@ gmail.com

Received May 05, 2011; Accepted November 21, 2011; Published December 07, 2011

Citation: Ningthoujam DS, Sanasam S, Nimaichand S (2011) Studies on Bioactive Actinomycetes in a Niche Biotope, Nambul River in Manipur, India. J Microbial Biochem Technol S6:001. doi:10.4172/1948-5948.S6-001

Copyright: (c) 2011 Ningthoujam DS, et al. This is an open-access article distributed under the terms of the Creative Commons Attribution License, which permits unrestricted use, distribution, and reproduction in any medium, provided the original author and source are credited 
Citation: Ningthoujam DS, Sanasam S, Nimaichand S (2011) Studies on Bioactive Actinomycetes in a Niche Biotope, Nambul River in Manipur, India J Microbial Biochem Technol S6:001. doi:10.4172/1948-5948.S6-001

Page 2 of 6

[14] slants at $4^{\circ} \mathrm{C}$ and as spore suspensions on $20 \%$ (v/v) glycerol at -20${ }^{\circ} \mathrm{C}$ [15] for further studies.

\section{Antimicrobial assay}

Test organisms: The test bacteria used were the Gram positive organisms Staphylococcus aureus (MTCC 96), Micrococcus luteus (MTCC 106), and Bacillus subtilis (MTCC 121), and the Gram negative bacteria Escherichia coli (MTCC 739) and Pseudomonas species (DN1); and the test fungi used were Candida albicans (MTCC 227) and Aspergillus niger (MTCC 1344). All the reference strains were procured from Microbial Type Culture Collection (MTCC), Institute of Microbial Technology (IMTECH), Chandigarh, India except for DN1 [16] which is a strain isolated in our laboratory.

Initial antimicrobial assay of the putative actinomycete isolates was carried out using the cross-streak technique $[17,18]$. Actinomycete isolates which showed inhibition of $>50 \%$ against the test organisms in the primary screening were further subjected to secondary screening by Kirby Bauer method [19] against the above test organisms. The actinomycete strains showing positive antimicrobial activities were subjected to phenotypic and genotypic characterization.

\section{Biocontrol assay of the bioactive strains}

Fungal pathogens were procured from MTCC, Chandigarh except for LSMU1(procured from Life Science Department, Manipur University). The bioactive strains were tested for biocontrol activity by dual culture method [20] against the rice pathogens, Fusarium oxysporum MTCC 287, Pyricularia oryzae MTCC 1477, Curvularia oryzae MTCC 2605 and Bipolaris oryzae LSMU1.

\section{Phenotypic and genotypic characterization}

The various morphological, physiological and biochemical characterization tests were carried out using the standard procedures [21-24]. The micromorphologies of the spore chains and the spore surfaces of 14 days old culture grown on Streptomyces agar were determined using Carl Zeiss microscope (AxioScope A.1, Germany, magnification $600 \mathrm{X}$ ). The cultural properties of the strains were evaluated according to the guidelines of the International Streptomyces Project (ISP) as described by Shirling \& Gottlieb [24].

$16 \mathrm{~S}$ rDNA amplification and sequencing were carried out for the bioactive isolates (having an inhibition zone of more than $17 \mathrm{~mm}$ diameter against the test organisms) using the primers (8F, 5'-AGAGTTTGATCCTGGCTCAG-3'; 357F, 5' - CTCCTACGGGAGGCAGCAG-3'; 1100R，5'-GGGTTGCGCTCGTTG-3'; 1492R，5'-GGTTACCTTGTTACGACTT-3'). The $16 \mathrm{~S}$ rDNA sequences were submitted to EzTaxon server version 2.1 [25], which contain manually curated databases of type strains of prokaryotes, for sequence analysis. Related strains were selected for alignment by CLUSTAL W program and phylogenetic analyses were done according to the neighbour-joining method [26] using the MEGA version $4.1[27,28]$. To determine the support of each clade, bootstrap analysis was performed with 1000 replications [29].

\section{Results and Discussion}

\section{Isolation of actinomycetes}

A total of 156 actinomycetes were isolated from the Nambul River, of which 47 (NRB1-1 to NRB1-47) were from the bank, 69 (NRS1-1 to NRS1-69) from the river bed, and 40 (NRP1-1 to NRP1-40) from the aquatic rhizospheric samples.

\begin{tabular}{|c|c|c|c|c|c|c|c|}
\hline \multirow{3}{*}{ Test isolates } & \multicolumn{7}{|l|}{ Test organisms } \\
\hline & \multicolumn{3}{|c|}{ Gram positive bacteria } & \multicolumn{2}{|c|}{ Gram negative bacteria } & \multicolumn{2}{|l|}{ Yeast/fungi } \\
\hline & MTCC 96 & MTCC 106 & MTCC 121 & MTCC 739 & DN1* & MTCC 227 & MTCC 1344 \\
\hline & \multicolumn{7}{|c|}{ Inhibition zone (in mm diameter) } \\
\hline Standard antibiotic discs & Erythromycin 16 & Penicillin-G 18 & Amikacin 18 & Streptomycin 18 & Rifampicin 12 & Amphotericin-B 16 & Nystatin 13 \\
\hline NRB1-1 & - & $13 \pm 0.29$ & $16 \pm 0.76$ & - & - & - & - \\
\hline NRB1-9 & - & - & $15 \pm 0.29$ & - & - & - & $11 \pm 0.58$ \\
\hline NRB1-19 & 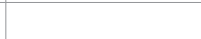 & $17 \pm 0.29$ & $18 \pm 0.76$ & $19 \pm 1.0$ & - & - & $15 \pm 0.76$ \\
\hline NRB1-20 & - & - & $15 \pm 0.58$ & - & - & - & - \\
\hline NRB1-25 & - & - & $16 \pm 1.0$ & - & - & - & - \\
\hline NRB1-29 & - & - & $15 \pm 0.58$ & $13 \pm 1.5$ & - & - & - \\
\hline NRB1-33 & - & - & $13 \pm 0.29$ & $16 \pm 0.58$ & - & - & - \\
\hline NRB1-44 & $16 \pm 1.0$ & - & $18 \pm 0.76$ & - & - & - & $17 \pm 1.0$ \\
\hline NRP1-5 & - & $13 \pm 0.58$ & $14 \pm 0.5$ & $11 \pm 0.76$ & - & - & - \\
\hline NRP1-13 & - & - & $18 \pm 1.0$ & - & - & - & $20 \pm 0.76$ \\
\hline NRP1-14 & - & - & - & - & - & $20 \pm 1.0$ & $21 \pm 0.58$ \\
\hline NRP1-18 & $21 \pm 0.58$ & $16 \pm 0.5$ & $15 \pm 0.76$ & - & - & - & $17 \pm 0.29$ \\
\hline NRP1-20 & - & - & $15 \pm 1.0$ & - & - & - & - \\
\hline NRP1-26 & $22 \pm 0.58$ & $15 \pm 0.29$ & $16 \pm 0.5$ & $18 \pm 1.0$ & - & $12 \pm 0.76$ & $18 \pm 0.76$ \\
\hline NRP1-28 & - & - & $16 \pm 0.29$ & - & - & - & - \\
\hline NRP1-29 & - & - & $15 \pm 0.76$ & - & - & - & - \\
\hline NRP1-35 & - & - & $18 \pm 0.5$ & $16 \pm 1.0$ & - & - & $18 \pm 0.76$ \\
\hline NRP1-40 & - & - & $13 \pm 1.0$ & - & - & - & - \\
\hline NRS1-1 & - & $12 \pm 1.5$ & $15 \pm 0.29$ & - & - & - & - \\
\hline NRS1-11b & $18 \pm 0.58$ & $20 \pm 0.29$ & $17 \pm 1.0$ & $18 \pm 0.76$ & - & - & - \\
\hline NRS1-18 & $13 \pm 0.58$ & - & $15 \pm 0.58$ & $17 \pm 0.29$ & - & - & - \\
\hline NRS1-30 & - & - & $14 \pm 1.0$ & - & - & - & - \\
\hline NRS1-39 & - & - & $16 \pm 0.58$ & $15 \pm 0.29$ & - & - & - \\
\hline
\end{tabular}

Table 1: Secondary Screening profile of the selected isolates exhibiting good antimicrobial activity in primary screening. 
Citation: Ningthoujam DS, Sanasam S, Nimaichand S (2011) Studies on Bioactive Actinomycetes in a Niche Biotope, Nambul River in Manipur, India. J Microbial Biochem Technol S6:001. doi:10.4172/1948-5948.S6-001

Page 3 of 6

\section{Antimicrobial assay}

Based on the results of primary screening, 23 strains (11.1\%) showed an inhibition zone of more than $50 \%$, against one or more of the test pathogens These isolates were then shortlisted for secondary screening (Table 1). Of 23 strains subjected to secondary screening, 9 (39.1\%) isolates (NRB1-19, NRB1-44, NRP1-13, NRP1-14, NRP1-18, NRP126, NRP1-35, NRS1-11b and NRS1-18) showed good antimicrobial activities with inhibition zone diameters of $17 \mathrm{~mm}$ or more against one or more of the test organisms. Among these bioactive isolates, 2 (NRS1$11 \mathrm{~b}$ and NRS1-18) were found to be purely antibacterial and 6 (NRB119, NRB1-44, NRP1-13, NRP1-18, NRP1-26 and NRP1-35) had broad antimicrobial activities. Interestingly, the strain NRP1-14 specifically showed potent antifungal activity against $C$. albicans and A. niger. None of the isolates was found to be bioactive against Pseudomonas aeruginosa. Reports of antimicrobial actinomycetes from freshwater habitats are rare. Elliah et al. [30] obtained 30 actinomycete isolates from sediments of Krishna River in Andhra Pradesh, India, of which 16 (53.3\%) exhibited excellent antagonistic properties in cross streak method. On detailed submerged fermentation studies, it was found that 12 isolates $(40.0 \%)$ had antibacterial and $9(30 \%)$ had antifungal activities. Five (16.6\%) isolates showed both antibacterial and antifungal activities. Singh et al. [31] isolated 37 actinomycetes from phoomdi (floating putrefying vegetation) in Loktak Lake in Manipur, India. Twentyone (56.7\%) isolates showed antimicrobial activities against test microorganisms in primary screening. Of these, 12 (32.4\%) were found to have broad spectrum (antibacterial and antifungal) activities.

\section{Biocontrol assay of the bioactive strains}

Three of the bioactive isolates, i.e NRP1-14, NRP1-18 and NRP1-26, showed antagonistic activity against one or more rice fungal pathogens [32]. These strains also exhibited phosphate solubilizing, siderophore, ammonia production and chitinase activities, showing their potential

\begin{tabular}{|l|l|l|l|l|l|}
\hline Test isolates & P solubilization & IAA & Siderophore & $\mathrm{NH}_{3}$ Production \\
\hline NRP1-14 & + & - & + & + & + \\
\hline NRP1-18 & + & + & + & + & + \\
\hline NRP1-26 & + & + & + & + \\
\hline
\end{tabular}

Table 2: Plant growth promoting characteristics of the antagonistic actinomycete isolates.

\begin{tabular}{|c|c|c|c|c|c|c|c|c|c|}
\hline Name of the test & NRB1-19 & NRB1-44 & NRP1-13 & NRP1-14 & NRP1-18 & NRP1-26 & NRP1-35 & NRS1-11B & NRS1-18 \\
\hline Gram's staining & + & + & + & + & + & + & + & + & + \\
\hline Production of diffusible pigment & - & - & - & - & - & - & - & - & - \\
\hline $\begin{array}{l}\text { Growth at } \\
4^{\circ} \mathrm{C} \\
15^{\circ} \mathrm{C} \\
25^{\circ} \mathrm{C} \\
30^{\circ} \mathrm{C} \\
37^{\circ} \mathrm{C} \\
42^{\circ} \mathrm{C} \\
60^{\circ} \mathrm{C}\end{array}$ & $\begin{array}{l}- \\
- \\
+ \\
+ \\
+ \\
- \\
-\end{array}$ & $\begin{array}{l}- \\
- \\
+ \\
+ \\
+ \\
+ \\
-\end{array}$ & $\begin{array}{l}- \\
- \\
+ \\
+ \\
+ \\
+ \\
-\end{array}$ & $\begin{array}{l}- \\
+ \\
+ \\
+ \\
+ \\
+ \\
-\end{array}$ & $\begin{array}{l}- \\
- \\
+ \\
+ \\
+ \\
+ \\
-\end{array}$ & $\begin{array}{l}- \\
- \\
+ \\
+ \\
+ \\
+ \\
-\end{array}$ & $\begin{array}{l}- \\
- \\
+ \\
+ \\
+ \\
+ \\
-\end{array}$ & $\begin{array}{l}- \\
- \\
+ \\
+ \\
+ \\
- \\
-\end{array}$ & $\begin{array}{l}- \\
- \\
+ \\
+ \\
+ \\
- \\
-\end{array}$ \\
\hline $\begin{array}{l}\text { Growth at } \mathrm{pH} \text { range } \\
5.2 \\
7.0 \\
8.0 \\
9.0 \\
10.0\end{array}$ & $\begin{array}{l}+ \\
+ \\
+ \\
+ \\
+\end{array}$ & $\begin{array}{l}+ \\
+ \\
+ \\
+ \\
+\end{array}$ & $\begin{array}{l}+ \\
+ \\
+ \\
+ \\
+\end{array}$ & $\begin{array}{l}+ \\
+ \\
+ \\
+ \\
+\end{array}$ & $\begin{array}{l}+ \\
+ \\
+ \\
+ \\
+\end{array}$ & $\begin{array}{l}+ \\
+ \\
+ \\
+ \\
+\end{array}$ & $\begin{array}{l}+ \\
+ \\
+ \\
W \\
-\end{array}$ & $\begin{array}{l}+ \\
+ \\
+ \\
+ \\
+\end{array}$ & $\begin{array}{l}- \\
+ \\
+ \\
+ \\
+\end{array}$ \\
\hline $\begin{array}{l}\text { Growth in the presence of } \\
2 \% \mathrm{NaCl} \\
5 \% \mathrm{NaCl} \\
7 \% \mathrm{NaCl} \\
10 \% \mathrm{NaCl}\end{array}$ & $\begin{array}{l}+ \\
+ \\
W \\
-\end{array}$ & $\begin{array}{l}+ \\
+ \\
+ \\
-\end{array}$ & $\begin{array}{l}+ \\
+ \\
+ \\
-\end{array}$ & $\begin{array}{l}+ \\
+ \\
+ \\
+\end{array}$ & $\begin{array}{l}+ \\
+ \\
+ \\
-\end{array}$ & $\begin{array}{l}+ \\
+ \\
+ \\
-\end{array}$ & $\begin{array}{l}+ \\
+ \\
+ \\
W\end{array}$ & $\begin{array}{l}+ \\
+ \\
+ \\
-\end{array}$ & $\begin{array}{l}- \\
- \\
-\end{array}$ \\
\hline $\begin{array}{l}\text { Degradation of } \\
\text { Adenine } 0.5 \% \\
\text { Guanine } 0.05 \% \\
\text { Tyrosine } 0.5 \% \\
\text { Xanthine } 0.4 \% \\
\end{array}$ & $\begin{array}{l}+ \\
- \\
- \\
-\end{array}$ & $\begin{array}{l}- \\
- \\
+ \\
-\end{array}$ & $\begin{array}{l}- \\
- \\
- \\
-\end{array}$ & $\begin{array}{l}- \\
- \\
+\end{array}$ & $\begin{array}{l}- \\
- \\
+ \\
-\end{array}$ & $\begin{array}{l}- \\
- \\
+ \\
-\end{array}$ & $\begin{array}{l}- \\
- \\
- \\
-\end{array}$ & $\begin{array}{l}+ \\
- \\
+ \\
+\end{array}$ & $\begin{array}{l}+ \\
- \\
- \\
-\end{array}$ \\
\hline $\begin{array}{l}\text { Hydrolysis of } \\
\text { Casein } \\
\text { Starch } \\
\text { Urea }\end{array}$ & $\begin{array}{l}+ \\
+ \\
-\end{array}$ & $\begin{array}{l}+ \\
+ \\
-\end{array}$ & $\begin{array}{l}+ \\
+ \\
+\end{array}$ & - & $\begin{array}{l}+ \\
+ \\
+\end{array}$ & $\begin{array}{l}+ \\
+ \\
+\end{array}$ & $\begin{array}{l}- \\
+ \\
-\end{array}$ & $\begin{array}{l}+ \\
+ \\
+\end{array}$ & $\begin{array}{l}+ \\
+ \\
-\end{array}$ \\
\hline $\begin{array}{l}\text { Biochemical tests } \\
\text { Catalase activity } \\
\text { Oxidase activity } \\
\text { Methyl Red (MR) } \\
\text { Voges Proskauer (VP) } \\
\text { Citrate utilization } \\
\text { Indole production } \\
\text { Nitrate reduction } \\
\text { Gelatin liquefaction } \\
\mathrm{H}_{2} \mathrm{~S} \text { production }\end{array}$ & $\begin{array}{l}+ \\
+ \\
+ \\
- \\
- \\
- \\
+ \\
- \\
-\end{array}$ & $\begin{array}{l}+ \\
- \\
- \\
- \\
+ \\
- \\
- \\
- \\
-\end{array}$ & $\begin{array}{l}+ \\
- \\
+ \\
- \\
- \\
- \\
+ \\
- \\
-\end{array}$ & $\begin{array}{l}- \\
- \\
- \\
- \\
+ \\
- \\
+ \\
- \\
-\end{array}$ & $\begin{array}{l}- \\
- \\
- \\
+ \\
- \\
- \\
+ \\
- \\
-\end{array}$ & $\begin{array}{l}- \\
- \\
- \\
+ \\
- \\
- \\
+ \\
- \\
-\end{array}$ & $\begin{array}{l}- \\
- \\
+ \\
- \\
- \\
- \\
+ \\
- \\
-\end{array}$ & $\begin{array}{l}+ \\
- \\
- \\
- \\
+ \\
- \\
+ \\
- \\
-\end{array}$ & $\begin{array}{l}- \\
- \\
- \\
- \\
- \\
- \\
- \\
- \\
-\end{array}$ \\
\hline
\end{tabular}

Table 3: Biochemical and physiological tests of the bioactive actinomycete isolates. 
Citation: Ningthoujam DS, Sanasam S, Nimaichand S (2011) Studies on Bioactive Actinomycetes in a Niche Biotope, Nambul River in Manipur, India J Microbial Biochem Technol S6:001. doi:10.4172/1948-5948.S6-001

Page 4 of 6

\begin{tabular}{|c|c|c|c|c|c|c|c|c|c|c|}
\hline Media & Isolate $\rightarrow$ & NRB1-19 & NRB1-44 & NRP1-13 & NRP1-14 & NRP1-18 & NRP1-26 & NRP1-35 & NRS1-11b & NRS1-18 \\
\hline \multirow{2}{*}{ ISP1 } & AM & Cream & Grey & Magenta & Off White & Cream & Cream & Grey & Sandal wood & Reddish \\
\hline & SM & Cream & Light Grey & Magenta & Cream & Cream & Cream & Grey & Cream & Reddish \\
\hline \multirow{2}{*}{ ISP2 } & AM & Pale cream & Cream & Magenta & Cream & Pale Cream & Pale Cream & Grey & Sandal wood & Reddish brown \\
\hline & SM & Cream & Light Yellow & Magenta & Light Yellow & Cream & Cream & Brown & Light Yellow & Reddish Brown \\
\hline \multirow{2}{*}{ ISP3 } & AM & Cream & Cream & P.G. & Pale Cream & Grey & Grey & Grey & Cream & Reddish \\
\hline & SM & Cream & Brown & P.G. & Cream & Grey & Grey & Brown & Cream & Reddish Brown \\
\hline \multirow{2}{*}{ ISP4 } & AM & Cream & Grey & P.G. & Off White & Grey & Grey & Grey & Sandalwood & P.G. \\
\hline & SM & Cream & Light Grey & P.G. & Light Grey & Grey & Grey & Brown & Cream & P.G. \\
\hline \multirow{2}{*}{ ISP5 } & AM & White & Off White & Light range & Off White & White & White & Grey & Cream & P.G. \\
\hline & SM & White & White & Light Orange & White & Cream & Cream & Grey & Cream & P.G. \\
\hline \multirow{2}{*}{ ISP6 } & AM & White & Pale Cream & Magenta & Cream & Pale Cream & Pale Cream & Grey & Cream & Reddish Brown \\
\hline & SM & Pale Cream & Pale Cream & Cream & Brown & Pale Cream & Pale Cream & Dark Brown & Cream & Reddish Brown \\
\hline \multirow{2}{*}{ ISP7 } & AM & Off White & Cream & P.G. & Light Grey & Grey & Grey & Grey & Sandal wood & P.G \\
\hline & SM & Off White & Light Grey & P.G. & Grey & Grey & Grey & Light grey & Cream & P.G \\
\hline \multirow{2}{*}{ SCNA } & AM & Off White & Grey & Light Orange & Grey & Grey & Grey & Grey & Off White & Orange \\
\hline & SM & Grey & Brown & Orange & White & Yellow & Yellow & Brown & Yellow & Brown \\
\hline \multirow{2}{*}{ SA } & AM & Cream & Cream & Magenta & Pale Cream & Cream & Cream & Grey & Sandal Wood & Reddish Brown \\
\hline & SM & Cream & Light Brown & Cream & Cream & Light Brown & Light Yellow & Black & Cream & Brown \\
\hline \multirow{2}{*}{ TSA } & AM & Pale Cream & Pale Cream & Pale Cream & Pale Cream & Pale Cream & Pale Cream & Off White & Cream & P.G. \\
\hline & SM & Pale Cream & Pale Cream & Pale Cream & Pale Cream & Pale Cream & Pale Cream & Light Grey & Pale Cream & P.G \\
\hline
\end{tabular}

ISP- International Streptomyces Project, SA - Streptomyces Agar, TSA- Tryptone Soya Agar

AM- Aerial mycelium, SM- Substrate mycelium

P.G. - Poor Growth

Table 4: Growth morphology on different ISP and other actinomycete specific media.
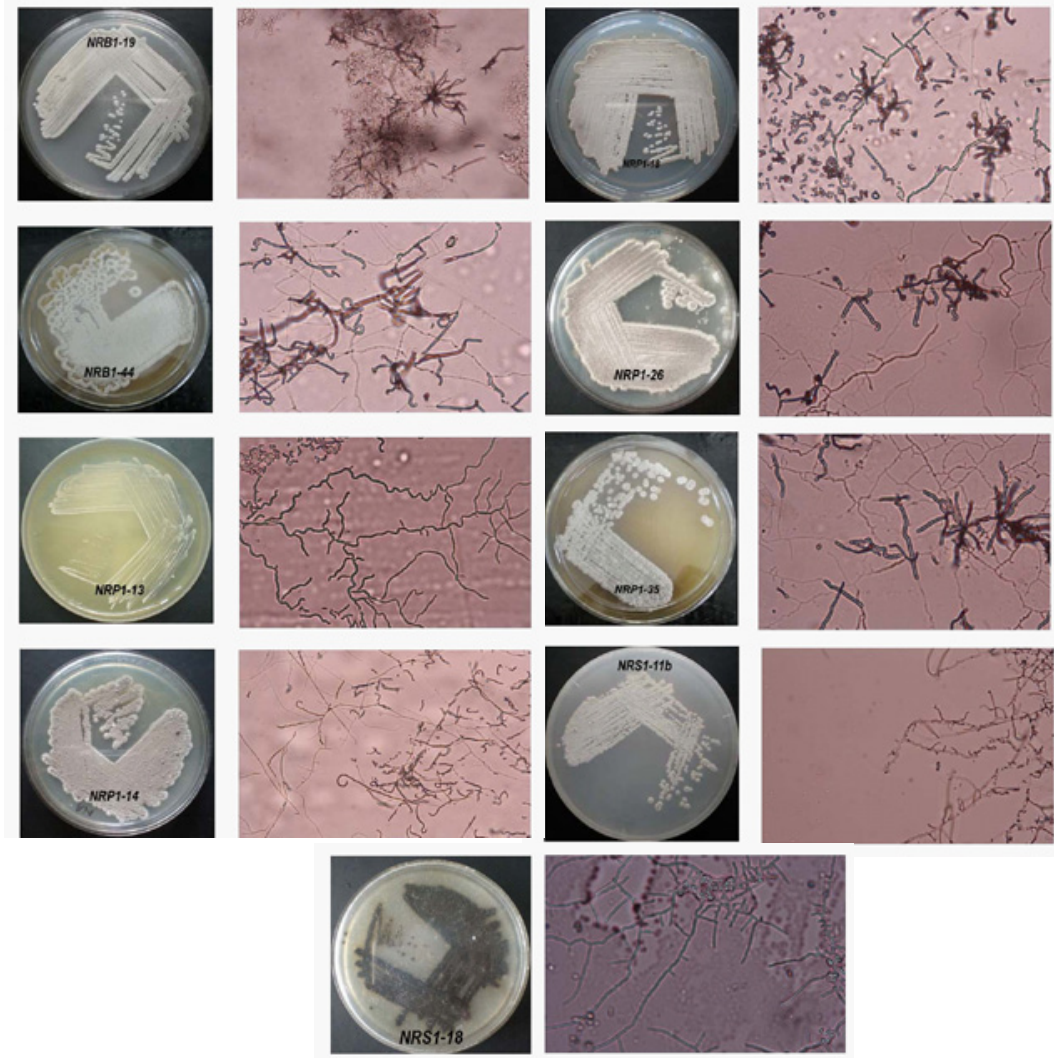

Figure 1: Morphological feature of the bioactive Nambul actinomycetes (NRB1-19, NRB1-44, NRP1-13, NRP1-14, NRP1-18, NRP1-26, NRP1-35, NRS1-11b, NRS118) and their micromorphologies. 
Citation: Ningthoujam DS, Sanasam S, Nimaichand S (2011) Studies on Bioactive Actinomycetes in a Niche Biotope, Nambul River in Manipur, India. J Microbial Biochem Technol S6:001. doi:10.4172/1948-5948.S6-001

Page 5 of 6

for plant growth promotion and biocontrol of pathogens (Table 2). These strains also had IAA producing abilities, with the exception of NRP1-14.

\section{Phenotypic and genotypic characterization}

Phenotypic characteristics of the bioactive strains and their growth morphologies on different ISP and other actinomycete specific media are shown in (Tables 3,4 .) The gross morphologies of the bioactive strains grown on SCNA media and their micromorphologies are shown in Figure 1.

NRP1-13 grew at $25-42^{\circ} \mathrm{C}, \mathrm{pH} 5.2-10$, and tolerated up to $7 \% \mathrm{NaCl}$ while NRS1-18 grew at $25-37^{\circ} \mathrm{C}, \mathrm{pH} 7-10$ and could tolerate $<2 \% \mathrm{NaCl}$. NRB1-19 and NRS1-11B grew well at $15-37^{\circ} \mathrm{C}, \mathrm{pH}$ 5.2-10 and tolerated up to $7 \% \mathrm{NaCl}$. Four isolates (NRB1-44, NRP1-18, NRP1-26 and NRP1-35) grew at $25-42^{\circ} \mathrm{C}, \mathrm{pH}$ 5.2-10 (though NRP1-35 grew poorly at $\mathrm{pH} 10$ ) and tolerated $2-7 \% \mathrm{NaCl}$ (NRP1-35 could grow even at $10 \%$ $\mathrm{NaCl})$. NRP1-14 grew well at $15-42^{\circ} \mathrm{C}, \mathrm{pH}$ 5.2-10 and tolerated $2-10 \%$ $\mathrm{NaCl}$. Most isolates were positive for casein as well as starch hydrolysis, except for NRP1-35, which was negative for casein hydrolysis, and NRP1-14, which was negative for both casein and starch hydrolysis.

Results of phylogenetic analyses (Figure 2) of the bioactive actinomycetes revealed that Streptomyces was the predominant actinomycete genus among the Nambul river strains, though Micromonospora and Nocardia were also recovered. Seven strains were identified as Streptomyces species. NRB1-19 was most closely related to Streptomyces parvus (similarity index 100\%), NRB1-44 to Streptomyces thinghirensis (similarity index 100\%), NRP1-14 to Streptomyces mutabilis (similarity index 99.805\%), NRP1-18 to Streptomyces subrutilis (similarity index 100\%), NRP1-26 to Streptomyces enissocaesilis (similarity index 99.728\%), NRP1-35 to Streptomyces drozdowiczii (similarity index 99.428\%) and NRS1-11b Streptomyces fragilis (similarity index 100\%). NRP1-13 was found to be most closely related to Nocardia asiatica (similarity index $99.780 \%$ ) and NRS1-18 to Micromonospora chalcea (similarity index 99.659\%).

Rifaat [33] reported the predominance of Streptomyces in water sample and that of Micromonospora in sediments of the Nile

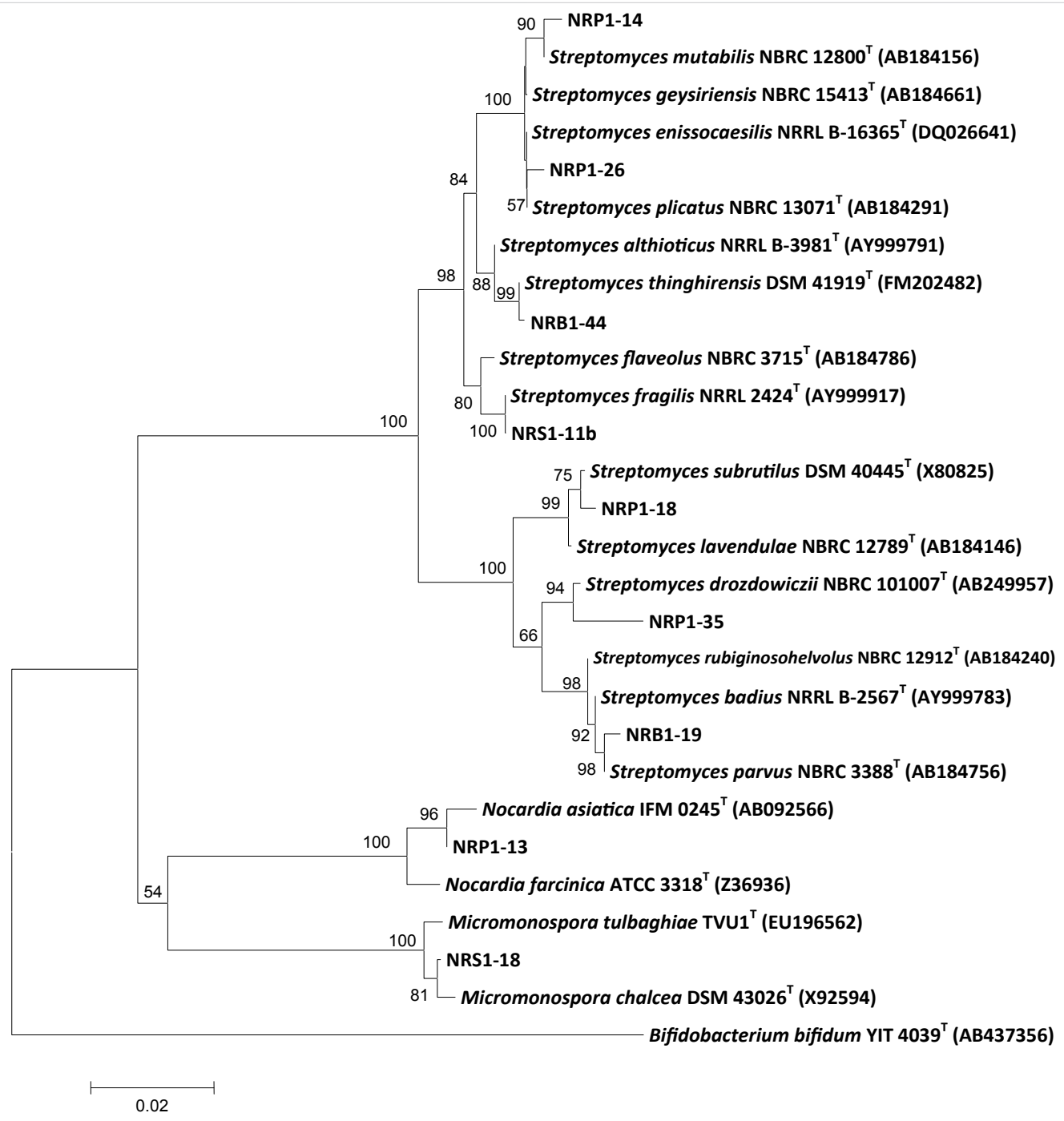

Figure 2: Phylogenetic relationship of the bioactive Nambul actinobacteria with its closest homolog. 
Citation: Ningthoujam DS, Sanasam S, Nimaichand S (2011) Studies on Bioactive Actinomycetes in a Niche Biotope, Nambul River in Manipur, India J Microbial Biochem Technol S6:001. doi:10.4172/1948-5948.S6-001

River. These Streptomyces strains were reported to have significant antimycotic activity. Elliah et al. [30] observed that Streptomyces strains, from Krishna river sediments in India, had significant antibacterial and antifungal activities. Our group had earlier showed potential for obtaining bioactive actinomycetes from niche habitats in Manipur including Nambul River [34]. The present study reemphasizes the promise of Nambul as source of antimicrobial actinomycetes. Although, freshwater habitats have been long ignored for actinomycete exploration, several recent reports corroborate the importance of such ecosystems for the search of antibiotic producing actinomycetes. A Streptomyces sp. AZ-NIOFD1, with broad-spectrum antimicrobial activity, was isolated from water sample of the Nile River in Egypt by Atta et al. [35]. Cwala et al. [36] reported Actinopolyspora sp. TR008, from Tyume River in South Africa which was active against both Gram positive and Gram negative bacteria. Sibanda et al. [37] recently stressed the significance of freshwater habitats as source of bioactive actinomycetes. They obtained actinomycete species belonging to Sachharopolyspora and Actinosynemma from Tyume River, South Africa. Crude extracts of these strains were found to exhibit potent antibacterial activity against both Gram positive and Gram negative bacteria.

Our preliminary findings showed promise of obtaining bioactive (antibacterial and antifungal) actinomycetes in an underexplored habitat, Nambul River in Manipur, India. Further studies on actinomycete population in the plethora of wetlands in Manipur-lakes, rivers, ponds, and marshes etc.- hold promise for obtaining novel strains, or even species, of bioactive actinomycetes.

\section{Acknowledgements}

The authors acknowledge the kind help of Dr. S. Shivaji, Scientist (Deputy Director), CCMB, Hyderabad and Dr. S. Mayilraj, Scientist, MTCC, IMTECH, Chandigarh for providing the DNA sequencing facility.

\section{References}

1. Strzelczyk E, Rouatt JW, Peterson EA (1969) Studies on Actinomycetes from Soils of Baffin Island. Arctic 22: 130-139.

2. Terkina IA, Parfenova VV, Ahn TS (2006) Antagonistic Activity of Actinomycetes of Lake Baikal. Appl Biochem Microbiol 42: 173-176.

3. Cross T (1981) Aquatic actinomycetes: a critical survey of the occurrence growth and role of actinomycetes in aquatic habitats. J Appl Bacteriol 50: $397-$ 423.

4. Okami $Y$ (1986) Marine microorganisms as a source of bioactive agents. Microbial Eco 12: 65-78.

5. Quadri LEN (2007) Drug Discovery Process of the $21^{\text {st }}$ Century. Infectious Disorders - Drug Targets 7: 230-237.

6. Goodfellow M, Fiedler H-P (2010) A guide to successful bioprospecting informed by actinobacterial systematics. Antonnie van Leeuwenhock 98: 119142.

7. Berdy J (2005) Bioactive microbial metabolites. J Antibiot 58: 1-26.

8. Rowbotha TJ, Cross T (1977) Ecology of Rhodococcus coprophilus and associated actinomycetes in freshwater and agricultural habitats. J Gen Microbiol 100: 231-240.

9. Williams ST, Shameemullah M, Watson ET, Mayfield Cl (1972) Studies on the ecology of actinomycetes in soil VI. The influence of moisture tension on growth and survival. Soil Biology and Biochem 4: 215-225.

10. Saadoun I, Al-momani F, Malkawi H, Mohammad MJ (1999) Isolation, identification and analysis of antibacterial activity of soil streptomycetes isolates from North Jordan. Microbios 100: 41- 46.

11. El-Nakeeb MA, Lechevalier HA (1963) Selective isolation of aerobic actinomycetes. Appl Microbiol 11: 75-77.

12. Kuster E, Williams ST (1964) Selection of media for isolation of streptomycetes Nature 202: 928-929.
13. Williams ST, Davies FL (1965) Use of antibiotics for selective isolation and enumeration of actinomycetes in soil. J Gen Microbiol 38: 251-261.

14. Jones KL (1949) Fresh isolates of actinomycetes in which the presence of sporogenous aerial mycelia is a fluctuating characteristic. J Bacteriol 57: 141145

15. Wellington EMH, Williams ST (1978) Preservation of actinomycete inoculum in frozen glycerol. Microbios Letters 6: 151-157.

16. Ningthoujam DS, Shovarani N (2008) Isolation and Characterization of a Pseudomonas aeruginosa Strain DN1 degrading p-Nitrophenol Research Journal of Microbiology 3: 345-351.

17. Oskay M, Tamer AU, Azeri C (2004) Antibacterial activity of some actinomycetes isolated from farming soils of Turkey. Afr J Biotechnol 39: 441-446.

18. Waksman SA (1961) The Actinomycetes: Classification, Identification and Description of Genera and Species. Baltimore: The Williams and Wilkins Company 2: 61-292.

19. Bauer AW, Kirb WM, Sherries JC, Turk M (1966) Antibiotic susceptibility testing by standard single disk method. Am J Clin Pathol 45: 493-496.

20. Hamdali H, Hafidi M, Virolle MJ, Ouhdouch Y (2008) Rock phosphate solubilizing Actinomycetes: screening for plant growth-promoting activities. World J Microbiol Biotechnol 24: 2565-2575.

21. Cappuccino JG, Sherman N (2004) Microbiology: A Laboratory Manual, Pearson Education (Singapore), Indian Branch. New Delhi.

22. Gunasekaran P (2000) Laboratory Manual in Microbiology. New Age International, New Delhi

23. MTCC, Actinomycetes-Lab Manual, IMTECH, Chandigarh 1998

24. Shirling EB, Gottlieb D (1966) Methods for characterization of Streptomyces species. Int J Syst Bacteriol 16: 313-340.

25. Chun J, Lee JH, Jung Y, Kim M, Kim S (2007) EzTaxon: a web-based tool for the identification of prokaryotes based on $16 \mathrm{~S}$ ribosomal RNA gene sequences. Int J Syst Evol Microbiol 57, 2259-2261.

26. Saitou N, Nei M (1987) The neighbor-joining method: A new method for reconstructing phylogenetic trees. Mol Biol Evol 4: 406-425.

27. Tamura K, Nei M, Kumar S (2004) Prospects for inferring very large phylogenies by using the neighbor-joining method. In Proceedings of the National Academy of Sciences (USA) 101: 11030-11035

28. Tamura K, Dudley J, Nei M, Kumar S (2007) MEGA4: Molecular Evolutionary Genetics Analysis (MEGA) software version 4.0. Mol Biol Evol 24: 1596-1599.

29. Felsenstein J. (1985) Confidence limits on phylogenies: An approach using the bootstrap. Evolution 39: 783-791

30. Elliah P, Raju KVV Bapi SN, Adinarayana K, Adinarayana et al. (2002) Bioactive actinomycets from Krishna river sediments of Andhra Pradesh. Hindusthan Antibiotics Bulletin 44: 1-4

31. Singh LS, Baruah I, Bora TC, (2006) Actinomycetes of Loktak habitat: Isolation and screening for antimicrobial activities. Biotechnology 5(2): 217-221.

32. Ningthoujam DS, Sanasam S, Tamreihao K, Nimaichand S (2009) Antagonistic activities of local actinomycete isolates against rice fungal pathogens. African Journal of Microbiology Research 3 (11): 737-742.

33. Rifaat HM (2003) The biodiversity of actinomycetes in the river Nile exhibiting antifungal activity. J Mediterranean Ecol. 4: 5-7.

34. Ningthoujam DS, Sanasam S, Nimaichand S (2009) Screening of Actinomycete Isolates from Niche Habitats in Manipur for Antibiotic Activity. American $J$ Biochem and Biotechnol 5 (4) : 221-225

35. Atta HM, Dabour SM, Desoukey SG (2009) Sparsomycin Antibiotic Production by Streptomyces Sp-NIOFD1: Taxonomy, Fermentation, Purification and Biological Activities. American-Eurasian J. Agri. \&Environ. Sci 5 (3): 368-377.

36. Cwala Z, Igbinosa EO, Oko Al (2011) Assessment of antibiotics production potentials in four actinomycetes isolated from aquatic environments of the Eastern Cape Province of South Africa. African J Pharmacy and Pharmocology 5 (2):118-124

37. Sibanda T, Mabinya V, Mazomba et al. (2010) Antibiotic Producing Potentials of Three Freshwater actinomycetes Isolated from the Eastern Cape Province of South Africa. Int J Mol.Sci 11 : 2612-2623. 